\title{
AMBIENTE DE APRENDIZAGEM PARA APOIAR O PROCESSO DE DESENVOLVIMENTO DO PRODUTO
}

Leticia Francischini Rodrigues (leticia.rodrigues@scpm.unimep.br) - Laboratório de Sistemas Computacionais para Projeto e Manufatura, Universidade Metodista de Piracicaba

Júlia de Andrade Bertazzi (julia.bertazzi@scpm.unimep.br) - Laboratório de Sistemas Computacionais para Projeto e Manufatura, Universidade Metodista de Piracicaba

Eduardo de Senzi Zancul (ezancul@usp.br) - Departamento de Engenharia de Produção da Escola Politécnica, Universidade de São Paulo

Klaus Schützer (schuetzer@scpm.unimep.br) - Laboratório de Sistemas Computacionais para Projeto e Manufatura, Universidade Metodista de Piracicaba

\begin{abstract}
RESUMO
O competitivo mercado global cria desafios como a redução do ciclo de vida de produtos e alto nível de personalização com custos baixos. Nesse cenário, a qualificação dos recursos humanos é um fator de sucesso na competição global. Para cumprir este requisito, foi desenvolvida uma nova maneira de aprender que integra a educação em um ambiente de fábrica real. Este conceito é chamado de Fábrica Ensino. Isso traz um ambiente que encoraja a aprendizagem experimental, simulando as características-chave de um ambiente de produção real em um ambiente acadêmico. Esta estratégia de ensino reduz a lacuna entre o conhecimento (universidade) e a ação (indústria). Este artigo descreve um ambiente de Fábrica Ensino que apoia o processo de desenvolvimento do produto implementado na Universidade Metodista de Piracicaba, Brasil. Este novo ambiente de aprendizagem simula um processo real de desenvolvimento de produtos para os programas de graduação e pósgraduação. A aplicação da Fábrica Ensino mostra as ferramentas aplicadas no processo de desenvolvimento do produto e envolve equipes de projeto, gerenciamento de produtos, solicitação de mudanças de engenharia e, durante todo o processo, os recursos de comunicação o fluxo de trabalho das ferramentas de gerenciamento de dados do produto usadas na implementação do ambiente.
\end{abstract}

Palavras-chave: Fábrica Ensino; Processo de Desenvolvimento do Produto; Desenvolvimento por Competências; Aprendizagem Experimental

Área: Gestão do Processo de Desenvolvimento de Produtos

\section{INTRODUÇÃ̃O}

As fábricas ensino foram criadas para incentivar e desenvolver o aprendizado experimental sobre melhorias no processo de manufatura simulando um ambiente de fabricação real e diminuindo a lacuna entre teoria e prática (KREIMEIER et al., 2014). O termo fábrica ensino está relacionado a projetos interdisciplinares em parceria com a indústria. Este modelo enfatiza a experiência prática de aplicação do conhecimento teórico para resolver problemas reais na indústria e no desenvolvimento de produtos. Recentemente, as fábricas ensino estão se tornando populares, possuem tamanhos variados, são sofisticadas e tem o objetivo de melhorar a experiência de aprendizagem (ABELE et al., 2015).

A introdução de conceitos de fábrica no ambiente acadêmico está relacionada ao lado prático do processo de ensino de engenharia, equilibrando a experiência em projeto, teoria 
relacionada a manufatura e sistemas de produção no processo de desenvolvimento do produto (PDP) (LAMANCUSA et al., 2001). Com esses objetivos, as fábricas ensino estão alinhadas com os requisitos da indústria para uma resposta rápida aos desafios do mercado, ao ciclo de vida do produto reduzido e aos produtos personalizados (TISCH et al., 2013).

A crescente demanda por soluções personalizadas requer conceitos inovadores e soluções para sistemas de manufatura. Processos flexíveis, tecnologias ágeis e sistemas de manufatura flexíveis são cada vez mais necessários e relevantes na indústria, aumentando a diversidade das fábricas ensino (WAGNER et al., 2012).

Portanto, o objetivo deste trabalho é apresentar o ambiente da Fábrica Ensino implementado pelo Laboratório de Sistemas Computacionais para Projeto e Manufatura (SCPM) na Universidade Metodista de Piracicaba (UNIMEP) no Brasil. A Fábrica Ensino da UNIMEP é um ambiente de aprendizagem sobre o processo de desenvolvimento de produtos que simula um processo real de desenvolvimento do produto para os programas de graduação e pósgraduação, combinando aprendizagem teórica e prática. O caso de uso da UNIMEP mostra que a aplicação do desenvolvimento de produtos e do processo de manufatura em cenários práticos diminui a lacuna entre a indústria e a academia.

O artigo está estruturado em quatro seções. Após o tópico de introdução na Seção 1, a Seção 2 fornece uma visão geral da Fábrica Ensino implementada. Na Seção 3, é apresentada a abordagem de desenvolvimento de produto utilizada no SCPM. A conclusão e a perspectiva de novas necessidades de pesquisa são discutidas na Seção 4.

\section{FÁBRICA ENSINO PARA O DESENVOLVIMENTO DO PRODUTO}

\subsection{Motivação}

Devido às crises econômicas que ocorreram nos últimos anos, há uma tendência para a reindustrialização das grandes economias mundiais. Nesse sentido, países desenvolvidos procuram qualificar sua força de trabalho para trabalhar em novos ambientes industriais, onde os produtos e os sistemas de produção sofrem mudanças rápidas e constantes. Assim, é fundamental que estudantes de engenharia sejam introduzidos rapidamente a esses conceitos. As universidades precisam procurar uma nova maneira de equilibrar a educação teórica de engenharia e o ensino prático, proporcionando aos estudantes uma curva de aprendizado mais rápida (LAMANCUSA et al., 2008). Através da fábrica ensino é possível trazer ambientes industriais para a universidade, preparando os novos engenheiros de forma mais eficaz para entrar no mercado de trabalho (MATT et al., 2014).

As fábricas ensino estão sendo desenvolvidas para transmitir conhecimento de processos e métodos aos estudantes e profissionais da indústria, através da simulação de um ambiente de produção real (KREIMEIER et al., 2014). Existem várias iniciativas de fábricas ensino no mundo. Uma breve revisão da literatura sobre o assunto permite destacar o Centro de Produtividade Industrial (CiP), implementado pelo PTW na TU Darmstadt, como uma das fábricas ensino pioneiras nesta área (CACHAY; ABELE, 2012); a Fábrica Ensino para Engenharia Industrial Avançada (aIE) no Instituto de Manufatura e Gestão Industrial (IFF) da Universidade de Stuttgart; IFactory, na Universidade de Windsor, Canadá (WAGNER et al., 2012); e a fábrica ensino com foco em PLM (gerenciamento do ciclo de vida do produto) na Universidade EAFIT na Colômbia (MORA-OROZCO et al., 2015).

Ao analisar as fábricas estudadas, observa-se que as fábricas ensino implementadas têm foco nos vários aspectos da produção, a partir de um produto já estruturado para manufatura em sistemas de fabricação flexíveis. No entanto, as empresas precisam de treinamento 
multidisciplinar, incluindo aqui todas as etapas do ciclo de desenvolvimento do produto e suas ferramentas. Nesse sentido, também é necessário configurar ambientes industriais de aprendizado que atuem nos estágios anteriores à realização do produto, focando o ciclo de desenvolvimento do produto e suas ferramentas, como PDM, CAD, CAM, CAE, etc.

Essa foi a motivação para o SCPM criar um ambiente industrial de aprendizado focado no processo de desenvolvimento do produto. Este ambiente deve oferecer todas as ferramentas digitais aplicadas no processo de desenvolvimento do produto, incluindo seu gerenciamento com os recursos disponíveis em um sistema PDM.

\subsection{Breve histórico sobre o SCPM}

A Fábrica Ensino para o Processo de Desenvolvimento do Produto foi desenvolvida e implementada pelo Laboratório de Sistemas Computacionais para Projeto e Manufatura (SCPM) da Universidade Metodista de Piracicaba (UNIMEP).

O laboratório foi criado há mais de 20 anos e conta com uma equipe de pesquisadores em tempo integral composta por um professor titular, doutorandos, mestrandos, estudantes de graduação e equipe de suporte técnico. O laboratório desenvolve projetos de pesquisa próximos de indústrias que permitam uma rápida implementação dos resultados tecnológicos obtidos. Para essas atividades, o laboratório possui modernos recursos de hardware e software.

O SCPM, procurando atender às novas necessidades de empresas de pequeno e médio porte, começou a trabalhar no Gerenciamento do Ciclo de Vida do Produto. Hoje, possui um ambiente de desenvolvimento do produto com as características de uma indústria, denominado de Fábrica Ensino para o Processo de Desenvolvimento do Produto, realizando atividades de desenvolvimento de produtos e simulações no processo de gerenciamento de dados do produto envolvendo todo o ciclo de desenvolvimento do produto.

\subsection{O ambiente de ensino}

A Fábrica Ensino para o Processo de Desenvolvimento do Produto possui uma infraestrutura composta por estações de trabalho com sistemas CAD / CAM / CAE integrados a um ambiente de gerenciamento de ciclo de vida do produto (PLM).

As atividades da fábrica ensino são desenvolvidas durante as aulas das disciplinas de Desenvolvimento do Produto e Gestão do Produto ministradas para os estudantes de graduação e pós-graduação dos cursos de engenharia da Universidade Metodista de Piracicaba. $\mathrm{O}$ ambiente de trabalho utiliza diferentes ferramentas para as atividades de desenvolvimento do produto, como: o software Teamcenter ${ }^{\circledR}$, que é uma das principais ferramentas disponíveis no mercado para solução de gerenciamento de dados de produtos; Sistema $\mathrm{NX}^{\circledR}$ que permite a integração do projeto do produto, engenharia e manufatura, associando funções CAD, CAE e CAM, apoiando os projetistas no desenvolvimento de componentes, montagem de subconjuntos e produto final e simulações; Microsoft ${ }^{\circledR}$ Office, auxilia na documentação dos processos de construção e nas especificações dos componentes e montagens adotados pelo supervisor e pelos projetistas.

São realizadas, semestralmente, aulas de desenvolvimento e gerenciamento de produtos. As aulas possuem 30 alunos os quais são divididos em seis equipes de projeto. Cada uma dessas equipes é composta por cinco estudantes, sendo quatro projetista e um supervisor. O papel de cada participante da equipe é decidido pelo próprio grupo. Decidida esta divisão, as funções são formalizadas usando os recursos do software Teamcenter ${ }^{\circledR}$. Cada estudante recebe o direito de acesso exigidos para a sua função. 
Neste ambiente, é responsabilidade do supervisor gerenciar o ciclo de desenvolvimento do produto; criar os itens a serem projetados; distribuir as tarefas individuais e encaminhar para cada projetista os componentes a serem construídos. O supervisor também é responsável por revisar o componente construído, liberando os componentes aprovados. Posteriormente, seguem-se os mesmos passos para a montagem dos subconjuntos e, finalmente, para a montagem do produto final. Os projetistas são responsáveis por projetar os componentes solicitados; enviar os componentes projetados para revisão; realizar as modificações necessárias dos componentes reprovados; realizar as simulações necessárias; montagem dos subconjuntos e do produto final.

\subsection{Descrição do produto}

O produto utilizado no treinamento é um eixo de cruzeta, o qual possui 11 diferentes componentes, agrupados em 3 subconjuntos que compõem o produto final, como pode ser observado na Figura 1.

Este produto foi escolhido em função de sua média complexidade, além de ser necessária a realização de simulações cinemáticas para que possíveis interferências sejam observadas e corrigidas. Com este produto, os estudantes podem utilizar os conhecimentos prévios adquiridos ao longo da vida acadêmica, ao aplicarem habilidades de construção de peças em um software CAD.

O subconjunto 1 é formado por 5 componentes; o subconjunto 2 consiste em 3 componentes; e o subconjunto 3 é composto por 2 componentes. A montagem desses três subconjuntos e a inserção de um pino de fixação resulta na montagem do conjunto completo do produto.

O processo de construção bottom-up foi adotado para esse ambiente de ensino, no qual cada componente é construído baseado em desenhos preparados pelo time e a partir disto, os subconjuntos e conjunto final são realizados. A construção de cada componente é auxiliada pelo software Siemens NX ${ }^{\circledR}$ CAD.

Cada um dos subconjuntos deve ser simulado, utilizando o software $\mathrm{NX}^{\circledR} \mathrm{CAE}$, considerando a cinemática do produto, para verificar quaisquer interferências e colisões operacionais, gerando os pedidos de alteração do produto quando necessário.

Figura 1. Eixo de Cruzeta.

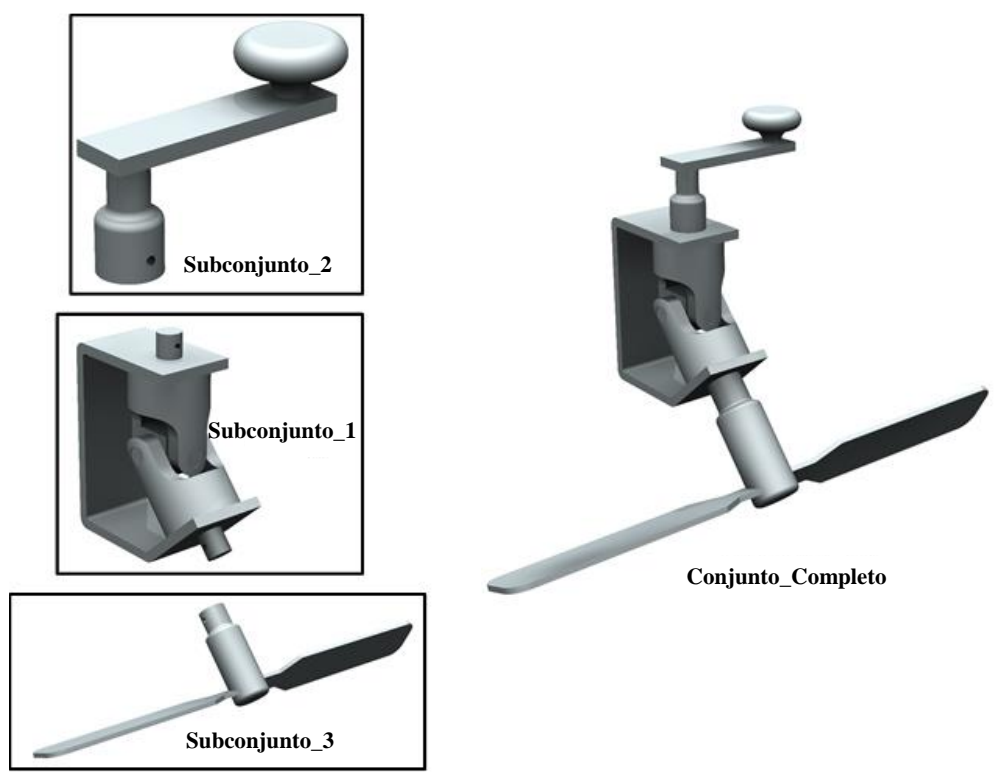

Fonte: Elaborada pelos autores. 
No treinamento, os estudantes devem desenvolver dois produtos que possuem componentes comuns e componentes distintos, sendo que estes são designados como produto 1 e produto 2 . Inicialmente, o subconjunto 1 do produto 1 é projetado considerando que as junções do eixocubo são feitas por interferência. Para o subconjunto 1 do produto 2, é solicitado que a junção eixo-cubo seja modificada para uma união com chaveta, dessa forma, o produto 2 será construído usando componentes do produto 1 e novos componentes. Também deve ser considerado que um dos componentes do produto 2 é desenvolvido por um fornecedor externo, portanto, é necessário importar esse componente para o Teamcenter ${ }^{\circledR}$.

Quando o produto 2 é finalizado, é possível verificar as inter-relações entre componentes e subconjuntos nos dois produtos construídos. Com isso, os projetistas podem ter a informação sobre a interação entre os componentes sempre que uma solicitação de alteração de um componente for recebida. Este procedimento, chamado de análise de impacto, é implementado nesta fábrica ensino e é apresentado nas seções a seguir. A função de análise de impacto ajuda o projetista a identificar se um componente que será modificado pertence a mais de um produto e como as mudanças solicitadas podem afetar todos os produtos envolvidos.

O ambiente para o desenvolvimento do produto também deve considerar o direito de acesso para outros usuários, como por exemplo, o departamento de planejamento de processos em um cenário de engenharia simultânea, para que estes possam visualizar o produto em desenvolvimento. Para que isso aconteça sem a necessidade de que cada usuário também tenha uma licença do sistema CAD utilizado, é necessário, além dos modelos geométricos específicos do sistema CAD, a geração de um modelo 3D neutro no formato JT, permitindo que qualquer usuário com direito de acesso ao sistema PDM possa visualizar o produto em desenvolvimento. Desta forma, é possível compartilhar informações entre diferentes áreas ao longo do processo de desenvolvimento do produto, o que permite que todas as áreas envolvidas no ciclo de vida do produto colaborem na realização do projeto.

O software, utilizado neste ambiente de ensino também fornece a ferramenta lista de materiais (Bill Of Materials - BOM), que compreende os componentes e a estrutura do produto informações fundamentais de engenharia, uma vez que na lista de materiais é registrada a estrutura do produto e suas informações correspondentes. A lista de materiais contribui para a integração departamental porque a informação é compartilhada pelos departamentos envolvidos no processo. Além disso, ela também pode conter outras informações, como o plano de processo, ferramentas e acessórios necessários para apoiar o processo de fabricação (ELMARAGHY et al., 2013).

\subsection{Gerenciamento do processo de desenvolvimento do produto}

Todas as atividades desenvolvidas com o auxílio de uma ferramenta de gerenciamento de dados do produto exigem um fluxo de trabalho (workflow) que oriente o processo. Um fluxo de trabalho pode ser definido como qualquer conjunto de atividades realizadas de forma coordenada, em série e/ou em paralelo, por indivíduos ou grupos com um objetivo comum (CHIRKIN; KOVALCHUK, 2014).

No ambiente da fábrica ensino, foram criados quatro fluxos de trabalho para diferentes situações de uso, todos têm a sequência básica de atividades mostradas na Figura 2.

O fluxo de trabalho inicia com a atividade "Início" onde o supervisor inicia o processo com a inclusão de um novo item e seu correspondente projetista no fluxo de trabalho, neste momento todas as instruções necessárias são incluídas para que o trabalho possa ser desenvolvido. A segunda atividade "Construção" refere-se à atividade executada pelos projetistas, sendo possível quatro alternativas diferentes referentes aos quatro tipos de fluxo 
de trabalho: construção de um componente; montagem de um subconjunto; importação de um componente; ou geração de um desenho técnico. Na terceira atividade "Revisão"; o componente desenvolvido pelo projetista na etapa anterior, é encaminhado ao supervisor para que este avalie o resultado da atividade "Construção". O supervisor possui duas possibilidades: no primeiro caso, uma falha do trabalho é identificada e, portanto, o processo retorna à fase de "Construção" e o projetista deve trabalhar novamente no componente, corrigindo os erros encontrados ou realizando as melhorias definidas pelo supervisor. No segundo caso, o componente é aprovado e o processo avança para a próxima atividade "Liberação", que significa que o componente foi aprovado e liberado. A próxima fase do fluxo de trabalho é "Conclusão", onde a tarefa é finalizada e quaisquer novas alterações no componente só podem ser criadas a partir uma nova versão.

Figura 2. Atividades básicas do fluxo de trabalho.

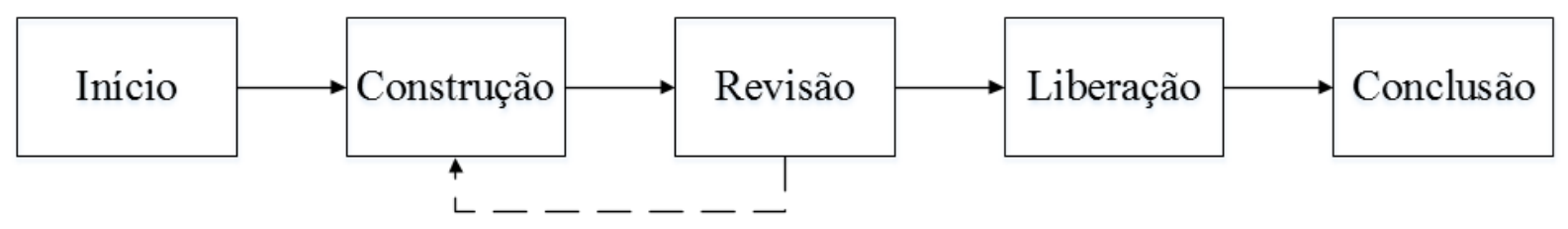

Fonte: Elaborada pelos autores.

O primeiro fluxo de trabalho a ser utilizado é para o processo de construção do componente. Portanto, o projetista deve construir o componente solicitado em um sistema CAD, integrado ao Teamcenter ${ }^{\circledR}$. O segundo fluxo de trabalho é para a montagem do subconjunto e para a montagem do produto final. O terceiro diz respeito à importação de componentes que foram desenvolvidos por um fornecedor externo e devem ser importados para o Teamcenter ${ }^{\circledR}$, desse modo estes componentes podem ser incluídos na estrutura final do produto. Por último, o quarto fluxo de trabalho é utilizado apenas após a aprovação da montagem final para a geração dos desenhos técnicos, para a documentação do produto.

Foi realizada a implementação de um quinto fluxo de trabalho, relacionado ao pedido de alteração (Enterprise Change Request - ECR) de um produto já finalizado e liberado. Devido à importância deste tópico, o assunto será apresentado em um tópico separado na seção 2.6.

\subsection{Pedido de alteração}

Um pedido de alteração do produto pode ocorrer no ciclo de vida do produto. Este pedido pode ser originado pelo uso do produto, possíveis falhas, ou devido a uma melhoria no processo de produção. Independentemente da origem de um pedido de alteração, o histórico completo dessa alteração (revisão, aprovação e implementação) deve ser gerenciado e documentado. Essas modificações ou melhorias são propostas e implementadas por um pedido de alteração (Enterprise Change Request - ECR). Na fábrica ensino, para o processo de desenvolvimento do produto, esta atividade foi implementada considerando uma alteração solicitada pelo gerente de manufatura.

O gerente de manufatura é um ator externo à equipe de desenvolvimento de produtos. Este ator tem a função de solicitar uma mudança, pois identificou algum erro, problema ou possibilidade de melhoria no produto desenvolvido.

A implementação desse processo ocorre em duas etapas. O fluxo de trabalho mostrado na Figura 3 apresenta a primeira etapa que consiste na solicitação de alteração de um componente feita pelo gerente de manufatura. $\mathrm{O}$ gerente de manufatura envia o pedido de alteração para o supervisor do produto, o qual analisa se o pedido é coerente ou não. Antes que qualquer decisão seja tomada, uma análise de impacto deve ser executada para identificar 
o impacto que a mudança no componente possa causar em todo o produto através das relações entre componentes, subconjuntos e conjunto final. Se o supervisor do produto aceitar o pedido de alteração, o fluxo de trabalho segue para a segunda etapa de implementação da alteração. Se o pedido de alteração sugerido pelo gerente de manufatura for rejeitado, uma notificação é enviada o informando sobre o motivo da recusa. Se a alteração for implementada, o gerente de manufatura também será notificado, ao término da segunda etapa, para estar ciente das mudanças que foram feitas.

Para a etapa de implementação da alteração, um novo fluxo de trabalho é iniciado, caracterizando a segunda etapa, como apresentado na Figura 4. Este fluxo de trabalho é utilizado apenas quando o pedido de alteração é aceito para implementação. Neste, o revisor envia o pedido de alteração para o projetista, o qual realiza as modificações no componente de acordo com o solicitado, criando, assim, uma nova versão para este componente. Ao final da atividade, o projetista envia o componente para o supervisor revisar a alteração. Se a alteração for aceita pelo supervisor, o fluxo de trabalho prosseguirá. Se a alteração for negada, o projetista deve fazer as melhorias solicitadas. Quando a alteração do componente está correta, a nova versão do produto é aprovada e finalizada.

Figura 3. Fluxo de trabalho para um pedido de alteração.

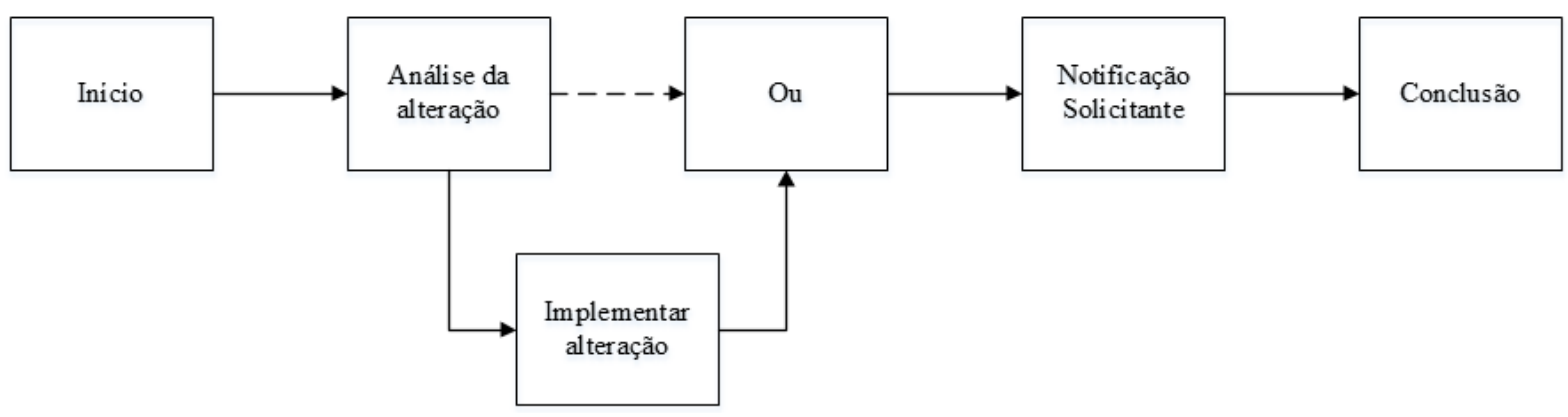

Fonte: Elaborada pelos autores.

Figura 4 - Fluxo de trabalho para implementação da alteração.

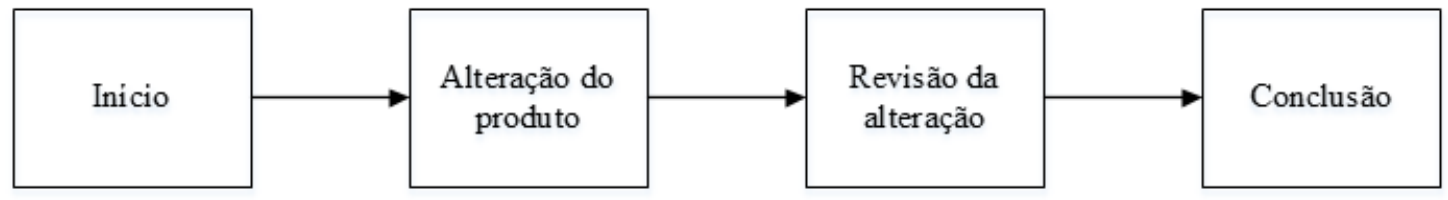

Fonte: Elaborada pelos autores.

\section{DESENVOLVIMENTO DO PRODUTO}

O treinamento na fábrica ensino é dividido em sete módulos: introdução ao sistema de ensino; criação e aprovação dos componentes (Criação do componente e Aprovação dos componentes); criação e gerenciamento de versões do componente (Gerenciamento de Versões); construção dos subconjuntos e do conjunto final (Montagem); criação de uma nova versão do produto (Novo produto); pedido para a alteração de um produto (Pedido de alteração). Estes módulos podem ser observados na Figura 5 e serão detalhados a seguir.

O processo de desenvolvimento do produto começa com o primeiro módulo de ensino, no qual a equipe se familiarizará com o sistema PLM, bem como com todo o ambiente de ensino em que o projeto será desenvolvido. Neste momento, os estudantes são divididos em equipes de projeto e são definidas as funções que cada um exercerá na equipe ao longo do processo de desenvolvimento do produto. O supervisor é o responsável por montar a estrutura do produto 
e criar os itens para cada componente, subconjunto e conjunto final, gerando um número de peça, ou código de identificação, para cada um desses componentes. Em seguida, as atividades para a construção do produto são distribuídas através de um fluxo de trabalho, conforme apresentado no tópico 2.4. As instruções e as especificações para a construção desses componentes também são enviadas por meio desse fluxo de trabalho através de um arquivo Office ${ }^{\circledR}$ Word, no qual é possível adicionar comentários e observações de todos os usuários envolvidos com a construção do componente.

Figura 5. Módulos do Processo.

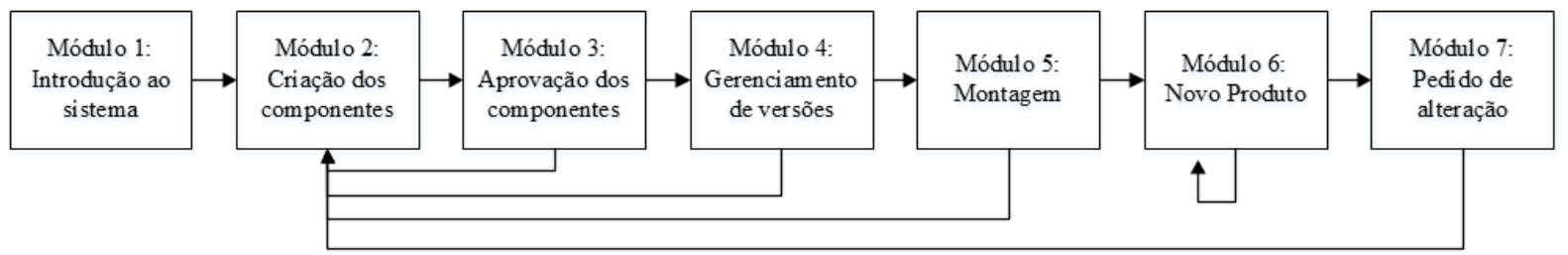

Fonte: Elaborada pelos autores.

No segundo módulo de ensino, os projetistas constroem os componentes de acordo com as instruções e especificações definidas no arquivo Office ${ }^{\circledR}$ Word e após a finalização da construção, o projetista dá continuidade ao fluxo de trabalho, enviando o componente ao supervisor.

No terceiro módulo, o supervisor revisa o componente de modo a confirmar que o mesmo não possui erro de construção e se ele foi construído de acordo com as especificações exigidas. Caso o componente esteja construído corretamente, ele é aprovado. Se o componente não estiver construído corretamente, o supervisor retorna o arquivo para o projetista responsável, notificando o erro. Desse modo o fluxo de trabalho retorna à etapa anterior de criação do componente. O componente será aprovado apenas quando for construído corretamente, de acordo com as especificações.

O quarto módulo de ensino orienta os alunos na criação de uma nova versão de um componente. Esta etapa ocorre quando um problema é identificado em um componente que já foi aprovado. Para que as correções sejam feitas, é necessário gerar uma nova versão do componente que será enviada ao projetista junto com os comentários e observações do supervisor sobre o problema identificado. Este passo ocorre durante o processo de desenvolvimento do produto e segue os mesmos procedimentos realizados do módulo 1 ao módulo 3. Após a realização da alteração pelo projetista, o componente é encaminhado para o supervisor para aprovação.

No quinto módulo, há a distribuição das atividades de montagem dos subconjuntos por meio do fluxo de trabalho, ou seja, cada subconjunto contendo o arquivo Office ${ }^{\circledR}$ Word com as instruções e as especificações são enviados para um projetista. Após a montagem, o subconjunto deve ser simulado considerando a cinemática do mecanismo. O processo de verificação e aprovação segue os mesmos procedimentos realizados nos módulos anteriores. Este processo é concluído com a montagem final do produto 1.

No sexto módulo de ensino, um caminho semelhante aos procedimentos anteriores é seguido, o produto 2 deve ser desenvolvido pela equipe como descrito no tópico 2.3. Com a aprovação dos produtos desenvolvidos e a geração da documentação técnica necessária para a produção, ambos os produtos são liberados para a produção.

O sétimo módulo refere-se a um pedido de alteração exigido através de um Enterprise Change Request, e se refere a identificação de um erro, problema ou possibilidade de melhoria no produto, observado por um gerente de manufatura durante o processo de 
manufatura. O erro pode ser identificado em qualquer componente de um produto final, portanto, tais melhorias serão implementadas em uma nova versão do componente identificado, ou subconjunto, quando necessário. O processo de solicitar uma alteração, implementar uma alteração e notificar ao solicitante é gerenciado através dos fluxos de trabalho apresentados no tópico 2.6. O gerente de manufatura faz um pedido formal de alteração e envia-o para o supervisor do produto que analisa a possibilidade de implantação e os impactos que esta alteração implicará na versão anterior do produto ou nos componentes. Se o supervisor do produto considerar que a melhoria não é válida, o pedido será negado. Caso contrário, a construção de uma nova versão do produto é iniciada. Ao final do ciclo, o gerente de manufatura é notificado da decisão tomada, bem como as consequentes implicações.

Esses sete módulos já foram implementados e se encontram em uso. Um oitavo módulo está em desenvolvimento e abrange a gestão do projeto dentro do sistema PDM, o que antes era realizado por outras ferramentas, como por exemplo MS Project ${ }^{\circledR}$.

Este módulo está considerando o uso do Schedule Manager ${ }^{\circledR}$ do Teamcenter ${ }^{\circledR}$. Esta ferramenta é utilizada para planejar e acompanhar o volume de horas de atividades alocada para cada projetista; permite administrar os recursos do processo (definindo responsáveis por cada etapa e tempo alocado), rastrear as entregas, assim como a conclusão de tarefas e atualização de informações; permite alterar recursos, datas do cronograma e trabalho estimado em tempo real. Uma vantagem importante desta ferramenta é o fato de que o planejamento e a execução dos projetos são gerenciados no mesmo sistema.

Basicamente todas as etapas descritas nos módulos anteriores seriam gerenciadas por meio da ferramenta de gerenciamento do projeto, em que cada componente estaria interligado pelo fluxo de trabalho dentro da ferramenta Schedule Manager ${ }^{\circledR}$. Sendo assim, todo o processo de análise e controle das tarefas são gerenciados por meio da mesma ferramenta. Além da possibilidade de melhor gerenciamento do tempo disponível para cada atividade de construção e revisão.

\section{CONCLUSÃO}

As fábricas ensino são uma estratégia de aprendizagem para diminuir a lacuna entre os trabalhos teóricos desenvolvidos nas universidades e os trabalhos práticos que ocorrem na indústria. Existem diferentes fábricas ensino em todo o mundo, como a CiP da Universidade Técnica de Darmstadt, a CC em Lyon, a LEP em Munique, etc. (ABELE et al., 2015). Cada fábrica ensino tem um foco e pesquisa diferentes. A proximidade da indústria local e a tendência global indicam o foco das fábricas ensino. No Brasil, existem poucas fábricas ensino concluídas ou desenvolvidas como a Cimatec do Senai na Bahia, a Fábrica do Futuro da Universidade de São Paulo e a Fábrica Ensino para o Processo de Desenvolvimento do Produto da Universidade Metodista de Piracicaba.

Este artigo apresentou a fábrica ensino implementada na Universidade Metodista de Piracicaba, no Brasil, que é um ambiente de desenvolvimento de produtos, onde os estudantes são apresentados aos desafios da modelagem de um produto. Os estudantes devem lidar com problemas reais de empresas, dividir o trabalho a ser feito, revisar o que já foi realizado, alterar alguns parâmetros e coordenar o processo de montagem.

Desta forma, o presente trabalho descreve as instalações da Fábrica Ensino para o Processo de Desenvolvimento do Produto da Universidade Metodista de Piracicaba e pode ser usado como orientação para a implementação de um teste de gestão de desenvolvimento de produto. 
Outras atividades de pesquisa se concentrarão na manufatura e projeto de componentes e produtos em um ambiente totalmente conectado. A evolução deste cenário está alinhada com a tendência global da Industrie 4.0 e como a conexão entre produtos e o ambiente de manufatura altera o projeto dos produtos, passando de processos de produção regulares para uma manufatura inteligente.

\section{REFERÊNCIAS}

ABELE, E.; et al. Learning factories for research, education, and training. Procedia CIRP, 32, 2015, p. 1-6.

CACHAY, J.; ABELE, E. Developing Competencies for Continuous Improvement Processes on the Shop Floor through Learning Factories-Conceptual Design and Empirical Validation. Procedia CIRP, 3, 2012, p. 638-643.

CHIRKIN, A.M.; KOVALCHUK, S.V. Towards Better Workflow Execution Time Estimation. IERI Procedia, 10, 2014, p. 216-223.

ELMARAGHY, H.; et al. Product variety management. CIRP Annals - Manufacturing Technology, 62, 2013, p. 629-652.

KREIMEIER, D.; et al. "Holistic learning factories - A concept to train lean management, resource efficiency as well as management and organization improvement skills". Procedia CIRP, 17, 2014, p. 184-188.

LAMANCUSA, J.S.; et al. The Learning Factory - Integrating design, manufacturing and business realities into engineering curricula - a sixth year report card. In: Proceedings of International Conference on Engineering Education, 2001, p. 1-6.

LAMANCUSA, J.S.; et al. The Learning Factory: Industry-Partnered Active Learning. Journal of Engineering Education, 97, 2008, p. 5-11.

MATT, D.T.; et al. Mini-factory - A learning factory concept for students and small and medium sized enterprises. Procedia CIRP, 17, 2014, p. 178-183.

MORA-OROZCO, J.; et al. PLM in a didactic environment: the path to smart factory. In: Proceedings of the 12th International Conference on Product Lifecycle Management, 2015.

TISCH, M.; et al. A Systematic Approach on Developing Action-oriented, Competency-based Learning Factories. Procedia CIRP, 7, 2013, p. 580-585.

WAGNER, U.; et al. The state-of-the-art and prospects of learning factories. Procedia CIRP, 3, 2012, p. 109-114. 